\title{
On action minimizing measures for the Monge-Kantorovich problem
}

\author{
Luca GRANIERI \\ Dipartimento di Matematica del Politecnico di Bari \\ via Amendola 126/B \\ 70126 Bari \\ Italy \\ e-mail: l.granieri@poliba.it
}

\begin{abstract}
In recent years different authors $([4,16,17])$ have noticed and investigated some analogy between Mather's theory of minimal measures in Lagrangian dynamic and the mass transportation (or Monge-Kantorovich) problem. We replace the closure and homological constraints of Mather's problem by boundary terms and we investigate the equivalence with the mass transportation problem. An Hamiltonian duality formula for the mass transportation and the equivalence with Brenier's formulation are also established.

2000 Mathematics Subject Classification: 37J50, 49Q20, 49 Q15.

Key words: Mather's minimal measures, Monge-Kantorovich problem, optimal transport problems, normal 1-currents.
\end{abstract}

\section{Introduction}

In the last ten years mass transportation problems, also known as MongeKantorovich problems, have been intensively studied in particular with respect to numerous and important applications and connections with other fields such as PDE, material sciences, probability and economics.

One of these connections, proposed by L.C. Evans in some recent works (see $[17,19])$, concerns Mather's theory of minimal measures in Lagrangian dynamic.

The Monge-Kantorovich and the Mather problem come from quite different settings and a connection between them is stated in [16] where the two problems are related by a careful formulation of both of them in terms of normal 1-currents, generalizing some results of Bangert who in [4] has shown that for the quadratic Lagrangian $L=|\cdot|^{2}$ Mather's problem of minimal measures can be formulated in 
terms of the following classical problem of geometric measure theory (see section 2 for definitions)

$$
\min \left\{M(T) \mid T \in \mathcal{N}_{1}^{c}(M),[T]=h\right\} .
$$

Actually, replacing the homological constraints in (1.1) by boundary terms we just obtain the Monge-Kantorovich problem with the geodesic distance as cost, see Proposition 3.2. Then, it is natural to think of a formulation of the MongeKantorovich problem in terms of probability measures on the phase space minimizing a suitable action functional. In the Mather problem the probability measures have to satisfy a closure and homological constraint (see section 1.2 for details). In the Monge-Kantorovich problem a suitable boundary condition has to be satisfied but, as we will see later, this is not always enough. The aim of this paper is just to investigate this formulation using some energy functionals defined on the space of normal 1-currents in the spirit of $[4,16]$.

Now, let us briefly describe the Monge-Kantorovich and the Mather problems and the main results of this paper in more details.

We will consider a compact Riemannian manifold $(M, g)$ without boundary and a Lagrangian $L: T M \rightarrow \mathbb{R}$ which satisfies the following properties:

(1) $L$ is regular (say $\mathcal{C}^{2}$ ) and positive,

(2) $L(x, \cdot)$ is superlinear in the fiber for all $x$,

(3) $L(x, \cdot)$ is strictly convex in the fiber for all $x$.

A standard assumption in Lagrangian dynamic is to consider Lagrangians $L$ which are $\mathcal{C}^{2}$-strictly convex, i.e. with Hessian $\frac{\partial^{2} L}{\partial v^{2}}(x, v)$ strictly positive definite as a quadratic form. However, in this paper we do not need this stronger condition on $L$. Actually, the $\mathcal{C}^{2}$ regularity assumption on the Lagrangian is needed just to have some differentiability properties of the Lax-Oleinik semigroup used in the proof of Theorem 4.1. For all the other purposes of the paper the $\mathcal{C}^{1}$ regularity is in fact enough.

Finally, $H: T^{*} M \rightarrow \mathbb{R}$ will denote the convex conjugate of $L$. The Lagrangian $L$ and the Hamiltonian $H$ are related by the Legendre transform $\mathcal{L}: T M \rightarrow T^{*} M$, defined by

and such that

$$
\mathcal{L}(x, v)=(x, \omega):=\left(x, \frac{\partial L}{\partial v}(x, v)\right),
$$

$$
H(x, \omega)=\langle\omega, v\rangle-L(x, v) .
$$

For an introduction to the Legendre transform and its properties we refer for example to [20].

\subsection{The Monge-Kantorovich problem}

Our description will be restricted to the setting of a compact Riemannian manifold $M$, however many of the concepts of this section could be formulated in general 
metric spaces or even in a topological setting. For a detailed presentation the interested reader may look at the book and lecture notes $[1,9,18,34,35]$, the paper [23] and for some of the applications [7, 8, 10, 11, 12, 13, 14, 27].

Let $c: M \times M \rightarrow \mathbb{R}^{+}$be a positive lower semicontinuous function. The Monge problem is formulated as follows: given two probability measures $f^{+}, f^{-}$ on $M$, find a map $t: M \rightarrow M$ such that $t_{\sharp} f^{+}=f^{-}(\sharp$ denotes the push-forward of measures) and such that $t$ minimizes

$$
\int_{M} c(x, t(x)) d f^{+}
$$

among the maps with the same property. It may happens that the set of admissible maps is empty (e.g. $f^{+}=\delta_{x}$ and $f^{-}=\frac{1}{2}\left(\delta_{y}+\delta_{z}\right)$ ). Moreover, because of the strong non-linearity, existence of minimizers is a difficult matter. In order to avoid this difficulties the problem can be reformulated in its Kantorovich's relaxation which amounts to:

$$
\min \left\{\int_{M \times M} c(x, y) d \gamma(x, y) \mid \gamma \in \mathcal{P}(M \times M), \pi_{\sharp}^{1} \gamma=f^{+}, \pi_{\sharp}^{2} \gamma=f^{-}\right\} .
$$

Where $\mathcal{P}$ stands for probability measures and $\pi^{1,2}$ are the projections on factors of $M \times M$. The admissible measures $\gamma$ for problem $\mathbf{M K}$ are called transport plans. Observe that if $t$ is admissible for the Monge problem then the measure $\gamma=(i d \times t)_{\sharp} f^{+}$is a transport plan for MK. Furthermore, the class of transport plans is never empty as it contains $f^{+} \otimes f^{-}$. The Kantorovich problem MK is linear and semicontinuity of $c$ is enough to prove existence of minimizers. Moreover this is true also if $c$ takes the value $+\infty$.

If $c$ is a distance then the cost of transportation

$$
d_{c}(\mu, \nu)=\min \left\{\int_{M \times M} c(x, y) d \gamma(x, y) \mid \pi_{\sharp}^{1} \gamma=f^{+}, \pi_{\sharp}^{2} \gamma=f^{-}\right\}
$$

defines a distance on $\mathcal{P}(M)$. If $d$ is the geodesic distance on the manifold then for $p \geq 1$ also

$$
d_{p}\left(f^{+}, f^{-}\right)=\left(\min \left\{\int_{M \times M} d^{p}(x, y) d \gamma(x, y) \mid \pi_{\sharp}^{1} \gamma=f^{+}, \pi_{\sharp}^{2} \gamma=f^{-}\right\}\right)^{1 / p}
$$

defines a distance on $\mathcal{P}(M)$ called Wasserstein distance, or $p$-Wasserstein distance. Moreover $\left(\mathcal{P}(M), d_{p}\right)$ is complete and $d_{p}$ metrizes the weak* convergence of measures. The metric structure induced on $\mathcal{P}(M)$ is extremely rich and will play an important role in the sequel.

In this paper we will always consider a "length" $\operatorname{cost} c(x, y)$, namely

$$
c(x, y)=\inf _{\gamma \in A C([0,1], M)}\left\{\int_{0}^{1} L(\gamma(t), \dot{\gamma}(t)) d t \mid \gamma(0)=x, \gamma(1)=y\right\} .
$$


In this case Brenier introduced a third formulation for the mass transportation problem (see $[5,9])$ : minimize

$$
K(\rho, E):=\int_{0}^{1} \int_{M} L(x, v(x, t)) d \rho_{t}(x) d t,
$$

among the pair $(\rho, E)$, with $\rho:[0,1] \rightarrow \mathcal{P}(M)$ a curve, $E_{t}$ a vectorial measure on $M$ defined $\rho_{t}$ almost everywhere, and $v(x, t)$ the Radon-Nikodym derivative of $E$ with respect to $\rho$, which satisfies the continuity equation:

$$
\left\{\begin{array}{l}
\frac{\partial \rho}{\partial t}+\nabla \cdot E=0 \quad \text { on }(0,1) \times M \\
\rho_{0}=f^{+}, \quad \rho_{1}=f^{-}
\end{array}\right.
$$

\subsection{Mather's minimal measures}

A measure $\mu \in \mathcal{M}(T M)$ is said to be closed if for all exact forms $\omega$ it results

$$
\int_{T M}\langle\omega(x), v\rangle d \mu=0 .
$$

According to this definition we set

$$
\mathcal{M}^{c}=\left\{\mu \in \mathcal{M}(T M) \mid \mu \text { is closed, } \mu \in \mathcal{P}(T M), \int_{T M} L(x, v) d \mu<+\infty\right\} .
$$

To each measure $\mu \in \mathcal{M}^{c}$ we can associate the homology class of $\mu$ which we will denote by $[\mu] \in H_{1}(M, \mathbb{R})$ (by duality with $\left.H^{1}\right)$. Indeed, thanks to the fact that $L$ is superlinear and $\int_{T M} L(x, v) d \mu<+\infty, \mu$ acts in a natural way on the set of the closed 1 -forms on $M$ by

$$
\omega \mapsto \int_{T M}\langle\omega(x), v\rangle d \mu
$$

and thanks to condition (1.4) this action passes to the quotient by the exact forms.

Once we fix an homology class $[h]$, Mather's variational problem amounts to:

$$
\min _{\mathcal{M}^{c}}\left\{\int_{T M} L(x, v) d \mu \mid[\mu]=[h]\right\} .
$$

We also define the action of $\mu$ by

$$
\mathcal{A}(\mu):=\int_{T M} L(x, v) d \mu .
$$

A remarkable property of problem $\mathbf{P}$ is the following: we minimize an action functional which depends on $L$ on measures which are merely closed. However, it turns out that the minimal measures are also invariant for the flow associated to the Lagrangian $L$, see for example $[4,15,21,28]$. 


\subsection{Description of this paper}

In [16] it is proved that Mather's problem $\mathbf{P}$ is equivalent to a dual formulation written in terms of currents. We will recall some essential facts from [16] in the next section. This formulation leads to a problem very similar to Brenier's formulation of the Monge-Kantorovich problem and, roughly speaking, it is obtained replacing the boundary constraints in (1.3) by a suitable homological constraint. So that, in order to relate problem $\mathbf{P}$ to the mass transportation one, it seems reasonable to replace the homological constraint by boundary terms. In particular we first investigate the following problem:

$$
\min \left\{\int_{T M} L(x, v) d \mu \mid \mu \in \mathcal{P}(T M), \partial p(\mu)=f^{+}-f^{-}\right\},
$$

where $f^{+}, f^{-} \in \mathcal{P}(M)$, and $p(\mu)$ is the current associated to $\mu$ by (1.5) (for the basic facts about currents we refer to the next section).

In section 3 we prove that problem $\mathbf{M}$ is equivalent to the Monge-Kantorovich one in the case $L(x, v)=g(v, v)^{\frac{1}{2}}$, see Proposition 3.2. Then we prove that for superlinear Lagrangians the convex set $\left\{\mu \in \mathcal{P}(T M) \mid \partial p(\mu)=f^{+}-f^{-}\right\}$is too big to describe the Monge-Kantorovich problem (see Example 3.6). Therefore, we have to consider an additional constraint which in general leads to a strict subfamily

$$
\Lambda \subset\left\{\mu \in \mathcal{P}(T M) \mid \partial p(\mu)=f^{+}-f^{-}\right\} .
$$

In Theorem 4.7 is in fact proved the following equality:

$$
\mathbf{M K}=\min \left\{\int_{T M} L(x, v) d \mu \mid \mu \in \Lambda\right\} .
$$

This result is obtained adding an extra "time" dimension, passing to a dual problem on currents as in [16] and then by a disintegration argument.

In order to make this strategy working, we establish an Hamiltonian duality formula for the transportation (Theorem 4.1) which on the other hand allow us to state the equivalence of Monge-Kantorovich problem and Brenier's formulation for cost associated to general Lagrangians. Finally, we show that in the case of $f^{+}=f^{-}$, in which case the current $p(\mu)$ is closed, the additional constraint $\Lambda$ does not play any role, see Lemma 4.10 , and requiring that $[p(\mu)]=[h]$ problem $\mathbf{M}$ reduces to the Mather problem $\mathbf{P}$. Finally, as a by-product of the study of problem M, we obtain a characterization of the Mañé critical value, see Proposition 4.11, which in our opinion deserves some attention by itself.

\section{Preliminary results}

\subsection{Normal 1-currents}

This section collects some definitions and technical facts about normal 1-currents and their representations which will be used explicitly in the paper or which are 
useful to give sense to some definitions. The exposition is adapted to the fact that the manifold $M$ is compact and then different from what would be on an open subset $\Omega$ of $\mathbb{R}^{N}$.

By $\Gamma^{\infty}\left(T^{*} M\right)$ we denote the space of $\mathcal{C}^{\infty}, 1$-dimensional forms. $\Gamma^{\infty}\left(T^{*} M\right)$ is usually equipped with the norm $\|\omega\|_{\infty}=\sup _{M}|\omega(x)|$. The space of normal, 1-dimensional currents is the space of linear, continuous functionals on $\Gamma^{\infty}\left(T^{*} M\right)$ and is denoted by $\mathcal{N}_{1}(M)$.

The subspace of the currents $T \in \mathcal{N}_{1}(M)$ such that $\langle T, \omega\rangle=0$ whenever $\omega$ is an exact form is the space of normal, closed 1-currents and it is denoted by $\mathcal{N}_{1}^{c}(M)$.

The mass norm of a normal current (in short: "mass") is defined as follows:

$$
M(T)=\sup \left\{T(\omega) \mid\|\omega\|_{\infty} \leq 1\right\}
$$

The boundary of a current is defined by duality with the differential through the formula:

$$
T(d \phi)=\partial T(\phi) \text { for all } \phi \in \mathcal{C}^{\infty}(M) .
$$

Then for a current $T$, being closed is equivalent to $\partial T=0$.

Any $T \in \mathcal{N}_{1}(M)$ can be represented by integration using a probability measure $\sigma$ on $M$ and a tangent vector field $X$ defined $\sigma$-a.e. as follows:

$$
T(\omega)=\int_{M}\langle\omega(x), X\rangle d \sigma
$$

in this case we briefly write $T=\sigma \wedge X$.

There are in the literature at least two canonical choices for the representation of a current. In line with [4] one can consider the following representation (which we denote by an underscript $s$ which stands for "special"):

$$
\sigma_{s}(B)=\frac{1}{M(T)} \sup \left\{T(\omega) \mid \omega \in \Gamma^{\infty}\left(T^{*} M\right),\|\omega\|_{\infty} \leq 1, \operatorname{spt}(\omega) \subset B\right\},
$$

for all open subsets $B$ of $M$, and $X_{s}$ is determined by the Radon-Nikodym Theorem and satisfies $g\left(X_{s}, X_{s}\right)=M(T)^{2} \sigma_{s}$-a.e.

There are many references in the literature for the representation of normal currents, among them we refer to [22] or Theorem 1 in section 2.3 of [24].

Normal currents are representable by integration and we point out that there are many possible choices for this representation. For example, given a representation $\sigma \wedge X$ of a current $T$, we can obtain another representation (which in special case will coincide with the first one) as follows: choose a function $c \in L_{\sigma}^{1}$ such that $\int_{M} c d \sigma=1$ and $c(x) \geq \delta>0$, then $\sigma_{1}=c(x) \sigma$ is a probability measure and $X_{1}=\frac{X}{c}$ is a vector field such that $\sigma_{1} \wedge X_{1}=T$.

Actually, the following lemma gives a more general vision of this last fact.

Lemma 2.1 Let $T=\sigma_{1} \wedge X_{1}$ and $T=\sigma_{2} \wedge X_{2}$. Then $\sigma_{i}<<\sigma_{j}$ and $X_{j}=\frac{\partial \sigma_{i}}{\partial \sigma_{j}} X_{i}$. 
In [16] Mather's problem is found to be equivalent to a dual formulation written in terms of currents. Actually, it turns out that the boundary terms do not play any role. So the equivalence found still holds simply replacing the homological constraint by boundary terms. For convenience we summarize some fundamental facts from [16]. In particular we introduce an energy functional on $\mathcal{N}_{1}$ associated to the Lagrangian $L$. As $L$ is not homogeneous, after some considerations one is convinced that the functional should depend not only on the current but also on the parametrization we choose for it. Therefore we define this energy functional by duality although some reader may prefer to take as the definition the formula given in Lemma 2.3.

For each $\sigma \in \mathcal{P}(M)$ and each $T \in \mathcal{N}_{1}(M)$ such that $\partial T=f^{+}-f^{-}$we define the following functional:

$$
\mathcal{L}(\sigma, T):=\sup _{\alpha, \omega} \int_{M} \alpha(x) d \sigma+T(\omega),
$$

where the sup is taken for $\alpha$ continuous function on $M$ and $\omega \in \Gamma^{\infty}\left(T^{*} M\right)$ which satisfy $\alpha(x)+H(x, \omega) \leq 0$ pointwise. Observe that this definition is related to the natural duality between $\mathcal{C}(M) \times \Gamma^{\infty}\left(T^{*} M\right)$ and $\mathcal{M}(M) \times \mathcal{N}_{1}(M)$.

Remark 2.2 Whenever $\mathcal{L}(\sigma, T)<+\infty$, an easy approximation argument allow to enlarge the class of admissible forms in (2.2) including forms with $L_{\sigma}^{\infty}$ coefficients. For more details see [16].

We have the following

Lemma 2.3 $\mathcal{L}(\sigma, T)$ is always positive and it is finite if and only if there exists a vector field $X \in L_{\sigma}^{1}(M)$ such that $T=\sigma \wedge X$. Moreover in such case we have

$$
\mathcal{L}(\sigma, T)=\int_{M} L(x, X(x)) d \sigma .
$$

At this point we can formulate the following problem on the space of currents:

$$
\min \left\{\mathcal{L}(\sigma, T) \mid \sigma \in \mathcal{M}(M), T \in \mathcal{N}_{1}(M), \partial T=f^{+}-f^{-}\right\} .
$$

We have the following

Theorem 2.4 The minimal values in problems $\boldsymbol{M}$ and (2.3) coincide.

\subsection{Duality}

In this section we recall some useful duality results for the Monge-Kantorovich problem. The following Theorem can be found in [35]. 
Theorem 2.5 Let $c(x, y)$ a positive continuous cost on $M$. Then the minimum value of the Kantorovich problem is equal to

$$
\sup _{\varphi, \psi \in \mathcal{C}(M, \mathbb{R})}\left\{\int_{M} \varphi(x) d f^{+}+\int_{M} \psi(y) d f^{-} \mid \varphi(x)+\psi(y) \leq c(x, y)\right\} .
$$

As noticed for example in [3], the statement of Theorem 2.5 is true also if the cost $c(x, y)$ is merely lower semicontinuous. In fact, one can pointwise approximate $c$ from below by an increasing sequence of continuous functions $c_{n}$. This is a standard fact in metric spaces, taking for example the Yosida transform:

$$
c_{n}=\inf \left\{c\left(x^{\prime}, y^{\prime}\right)+n d\left(x, x^{\prime}\right)+n d\left(y, y^{\prime}\right) \mid x^{\prime}, y^{\prime} \in M\right\} .
$$

If $\gamma_{n}$ are optimal plans for the transportation with the cost $c_{n}$ we can assume that $\gamma_{n} \stackrel{*}{\rightarrow} \gamma$ (Observe that $\gamma$ results a transport plan between $f^{+}$and $\left.f^{-}\right)$. Fixed $\delta>0$ and $m \leq n$, by $(2.4)$ we find $\varphi_{n}(x)+\psi_{n}(y) \leq c_{n}(x, y) \leq c(x, y)$ such that

$$
\int_{M \times M} c_{m} d \gamma_{n} \leq \int_{M \times M} c_{n} d \gamma_{n} \leq \int_{M} \varphi_{n} d f^{+}+\int_{M} \psi_{n} d f^{-}+\delta \leq \mathcal{D}+\delta .
$$

Where $\mathcal{D}$ is the value of the supremum in (2.4). Passing to the limit as $n \rightarrow+\infty$ we obtain $\int_{M \times M} c_{m} d \gamma \leq \mathcal{D}+\delta$.

Passing to the limit as $m \rightarrow+\infty$ and then letting $\delta \rightarrow 0^{+}$we conclude, since the opposite inequality is immediate.

If $c$ is the geodesic distance $d$ on $M$, duality formulation becomes:

Theorem 2.6 If $c(x, y)=d(x, y)$ then the minimum value of the Kantorovich problem is equal to

$$
\sup \left\{\int_{M} u d\left(f^{+}-f^{-}\right) \mid u \in \operatorname{Lip}_{1}(M, d)\right\} .
$$

Where $\operatorname{Lip}_{1}(M, d)$ is the set of Lipschitz functions with Lipschitz constant Lip $(u)$ not greater than 1. Actually, the supremum in Theorem 2.6 is attained and the optimal $u$ are called Kantorovich potentials.

For a function $\varphi: M \rightarrow \mathbb{R}$ we define the $c$-transform as follows:

$$
\varphi^{c}(x)=\inf \{c(y, x)-\varphi(y) \mid y \in M\} .
$$

We recall that if one consider $L^{1}(M, \mathbb{R})$ as set of admissible functions in Theorem 2.5 , then the supremum is always attained on couples of the form $\left(\varphi, \varphi^{c}\right)$, see for example [3].

In order to establish the duality formula of Theorem 4.1, we need to solve a time-dependent Hamilton-Jacobi equation. Although explicit solution (LaxOleinik formula) of this equation has been known from a long time, for reader's 
convenience we prefer to give here some notations and results since they could turn out not so easy to find in the literature, at least in the form we need.

For $t>0$ and $u \in \mathcal{C}(M, \mathbb{R})$, we define the Lax-Oleinik semigroup as:

$$
T_{t}^{-} u(x)=\inf _{\eta \in A C([0, t], M)}\left\{u(\eta(0))+\int_{0}^{t} L(\eta, \dot{\eta}) d s \mid \eta(t)=x\right\} .
$$

In the following Theorem we summarize some properties of this semigroup.

Theorem 2.7 In the previous assumptions we have:

(1) $\forall x \in M$ there exists an extremal curve $\gamma:[0, t] \rightarrow M$ with $\gamma(t)=x$ such that

$$
T_{t}^{-} u(x)=u(\gamma(0))+\int_{0}^{t} L(\gamma(s), \dot{\gamma}(s)) d s .
$$

Furthermore, such extremal $\gamma$ minimizes the action among all absolutely continuous curves $\sigma:[0, t] \rightarrow M$ with the same endpoints.

(2) There exists $K_{t}>0$ such that $T_{s}^{-} u$ is $K_{t}$-Lipschitz for every $s \geq t$.

(3) $\forall \varepsilon>0: \exists K_{\varepsilon}>0$ such that if $u, v \in \mathcal{C}(M, \mathbb{R})$ and $s, t \geq \varepsilon$ then

$$
\forall x \in M:\left|T_{s}^{-} u(x)-T_{t}^{-} v(x)\right| \leq\|u-v\|_{\infty}+K_{\varepsilon}|s-t| .
$$

(4) If $\gamma$ and $x$ are as in point (1) and if $T_{t}^{-} u$ is differentiable at $x$ then

$$
d_{x} T_{t}^{-} u(x)=\frac{\partial L}{\partial v}(\gamma(t), \dot{\gamma}(t))
$$

For a proof of these statements we refer to [15, 20, 21, 25].

Finally, we state a useful result of measure theory which will be used several times.

Theorem 2.8 (Disintegration) Let $X, Y$ locally compact and separable metric spaces, $f: X \rightarrow Y$ and $\mu \in \mathcal{M}(X)$. Then for $f_{\sharp} \mu-$ a.e. $y \in Y$ there exists $\mu_{y} \in \mathcal{P}(X)$ concentrated on $\{x \mid f(x)=y\}$ such that

(i) the map $y \mapsto \mu_{y}$ is Borel,

(ii) $\forall \varphi \in \mathcal{C}_{b}(X): \int_{X} \varphi(x) d \mu=\int_{Y}\left(\int_{X} \varphi(x) d \mu_{y}(x)\right) d f_{\sharp} \mu(y)$.

Moreover the measures $\mu_{y}$ are uniquely determined up to a negligible set with respect to $f_{\sharp} \mu$.

A proof of this assert can be found for example in [2], see also [1].

Remark 2.9 Denoting by $\sigma=f_{\sharp} \mu$, we will write $\mu=\mu_{y} \otimes \sigma$. In this paper we will use the above Disintegration Theorem in the particular case in which $f$ is a projection of a product space on one of his factors. Moreover, writing $\mu_{y} \otimes \sigma$ we will always assume that $\mu_{y}$ satisfy the condition (i) and (ii) of Theorem 2.8. 


\section{Comparisons between $\mathrm{M}$ and $\mathrm{MK}$}

The PDE's formulation of the Monge-Kantorovich problem is: minimize the functional

$$
K(\rho, E)=\int_{0}^{1} \int_{M} L(x, v(t, x)) d \rho_{t} d t
$$

among all maps $\rho:[0,1] \rightarrow \mathcal{P}(M)$ and $E:[0,1] \rightarrow \mathcal{M}(M, T M)$ which solve

$$
\left\{\begin{array}{l}
\frac{\partial \rho}{\partial t}+\nabla \cdot E=0 \quad \text { on }(0,1) \times M \\
\rho_{0}=f^{+}, \quad \rho_{1}=f^{-}
\end{array}\right.
$$

Where (3.1) is satisfied in the sense of distributions in $(0,1) \times M$ and $v(t, x)$ is the Radon-Nikodym derivative of $E_{t}$ with respect to $\rho_{t}$. Therefore, Brenier's formulation of the Monge-Kantorovich problem amounts to

$$
\min \{K(\rho, E) \mid(\rho, E) \text { solves }(3.1)\} \text {. }
$$

Observe that if $\varphi$ is a regular function on $M$, then (3.1) implies

$$
\frac{d}{d t}\left(\int_{M} \varphi(x) d \rho_{t}\right)=\int_{M} d \varphi(x) \cdot d E_{t}
$$

As in Lemma 2.3, one can easily check that whenever $K(\rho, E)<+\infty$, then $E$ is absolutely continuous with respect to $\rho$ and

$$
K(\rho, E)=\sup \left\{\int_{0}^{1} \int_{M} \alpha(t, x) d \rho_{t} d t+\int_{0}^{1} \int_{M} \beta(t, x) \cdot d E_{t} d t\right\} .
$$

Where the sup is taken for $\alpha, \beta$ continuous functions which satisfy the constraint $\alpha(t, x)+H(x, \beta(t, x)) \leq 0($ see $[9])$.

The equivalence between $\mathbf{B}$ and $\mathbf{M K}$ is well known for the quadratic Lagrangian $|v|^{2}$ (see for example [5]). Actually, this equivalence can be easily generalized to Lagrangian of the type $\varphi(|v|)$ (see [1,9]). Following the strategy of [9], we can also establish this equivalence for a general Lagrangian $L(x, v)$. We postpone this equivalence to section 4 .

We have a first comparison:

\section{Theorem 3.1 $M \leq B \leq M K$.}

Proof. Let $\eta$ be a Tonelli minimizer for the cost $c(a, b)$, and $\gamma$ an optimal plan for MK. Then we put: We claim that $(\rho, E)$ is admissible for $\mathbf{B}$. Indeed, if $\varphi$ is a test function we have

$$
\left[\frac{\partial \rho}{\partial t}+\nabla \cdot E\right](\varphi)=-\int_{M \times M} \int_{0}^{1}\left[\partial_{t} \varphi(t, \eta(t))+\left\langle d_{x} \varphi(t, \eta(t)), \dot{\eta}(t)\right\rangle\right] d t d \gamma(a, b)
$$




$$
=-\int_{M \times M} \int_{0}^{1} \frac{d}{d t}[\varphi(t, \eta(t))] d t d \gamma(a, b)=0 .
$$

Furthermore $\left\langle\rho_{0}, \varphi\right\rangle=\int_{M \times M} \varphi(a) d \gamma(a, b)=\left\langle f^{+}, \varphi\right\rangle$, and analogously $\rho_{1}=f^{-}$.

We can evaluate:

$$
\begin{aligned}
\mathbf{B} & \leq \sup _{\alpha, \beta} \int_{0}^{1} \int_{M}\left[\alpha(t, x) d \rho_{t}+\beta(t, x) \cdot d E_{t}\right] d t \\
& \leq \sup _{\beta} \int_{0}^{1} \int_{M}\left[-H(x, \beta(t, x)) d \rho_{t}+\beta(t, x) \cdot d E_{t}\right] d t \\
& =\sup _{\beta} \int_{M \times M} \int_{0}^{1}[-H(\eta(t), \beta(t, \eta(t))) d t d \gamma(a, b)+\langle\beta(t, \eta(t)), \dot{\eta}(t)\rangle d t d \gamma(a, b)] \\
& \leq \int_{M \times M} \int_{0}^{1} L(\eta(t), \dot{\eta}(t)) d t d \gamma(a, b)=\int_{M \times M} c(a, b) d \gamma(a, b)=\mathbf{M K} .
\end{aligned}
$$

For every $(\rho, E)$ admissible for $\mathbf{B}$, we consider the couple $(\sigma, T)$ defined by

$$
T(\omega)=\int_{0}^{1} \int_{M} \omega \cdot d E_{t} d t, \sigma=\int_{0}^{1} d \rho_{t} d t .
$$

By (3.2) we have

$$
\begin{aligned}
\partial T(\varphi)=T(d \varphi) & =\int_{0}^{1} \int_{M} d \varphi(x) \cdot d E_{t} d t=\int_{0}^{1} \frac{d}{d t}\left(\int_{M} \varphi(x) d \rho_{t}\right) d t \\
& =\rho_{1}(\varphi)-\rho_{0}(\varphi)=\left\langle f^{+}-f^{-}, \varphi\right\rangle .
\end{aligned}
$$

Hence $(\sigma, T)$ is admissible for problem (2.3).

Then, by Theorem 2.4 and the inequality $\mathcal{L}(\sigma, T) \leq K(\rho, E)$, we also obtain that $\mathbf{M} \leq \mathbf{B}$.

In [16] Mather's problem turns out to be equivalent to a formulation of the type of Brenier's problem with closed curves $\rho$ satisfying a suitable homological constraint. We remark that the arguments used in [16] do not work in this contest.

Let us consider the case of $L(x, v)=g(v, v)^{p}$ for $p \geq 1 / 2$. To point out the dependence on the Lagrangian we denote our problems by $\mathbf{M}_{p}, \mathbf{M K}_{p}, \mathbf{B}_{p}$. Let us check that Theorem 3.1 still holds for $p=1 / 2$, for which we have the geodesic distance $d(x, y)$ as cost for $\mathbf{M} \mathbf{K}_{1 / 2}$, while $\mathbf{M}_{1 / 2}$ is the minimum of the mass of 1-currents having $f^{+}-f^{-}$as boundary. Actually, we have the following

Proposition $3.2 M_{1 / 2}=B_{1 / 2}=M K_{1 / 2}$.

Proof. First let us observe that in this case we have $K(\rho, E)=\int_{0}^{1}\left\|E_{t}\right\| d t$, where $\left\|E_{t}\right\|$ is the total variation of $E_{t}$. Take $\gamma$ an optimal plan for $\mathbf{M K}_{1 / 2}$ and $(\rho, E)$ 
as in (3.3). Hence

$$
\begin{aligned}
\mathbf{B}_{1 / 2} & \leq \int_{0}^{1}\left\|E_{t}\right\| d t \leq \int_{M \times M} \int_{0}^{1}|\dot{\eta}(t)| d t d \gamma(a, b) \\
& =\int_{M \times M} d(a, b) d \gamma(a, b)=\mathbf{M K}_{1 / 2} .
\end{aligned}
$$

Consider $(\rho, E)$ admissible for $\mathbf{B}_{1 / 2}$ and put

$$
T(\omega)=\int_{0}^{1} \int_{M} \omega \cdot d E_{t} d t
$$

As in the proof of Theorem 3.1 we have that $\partial T=f^{+}-f^{-}$. We evaluate

$$
|T(\omega)| \leq \int_{0}^{1}\left\|E_{t}\right\| d t\|\omega\|_{\infty} \Rightarrow M(T) \leq \mathbf{B}_{1 / 2},
$$

from which we obtain $\mathbf{M}_{1 / 2} \leq \mathbf{B}_{1 / 2}$.

To check the opposite inequalities, let us consider a Kantorovich potential $u \in \operatorname{Lip}_{1}(M, d)$ as in Theorem 2.6.

Taking a current $T$ such that $\partial T=f^{+}-f^{-}$we have

$$
\mathbf{M K}_{1 / 2}=\int_{M} u d\left(f^{+}-f^{-}\right)=\partial T(u)=T(d u) \leq M(T) .
$$

Taking the minimum with respect to $T$ the statement follows.

Remark 3.3 Observe that the couple $(\rho, E)$ defined in (3.3) is optimal for $\mathbf{B}_{1 / 2}$ while the correspondent current $T$ defined by (3.4) is optimal for $\mathbf{M}_{1 / 2}$.

Remark 3.4 The equivalence between the three different problems stated in Proposition 3.2 is already known since in this case $\mathbf{M}_{1 / 2}$ can be seen as a shape optimization problem (see $[7,8]$ ). Indeed, in terms of vectorial measures, the value $\mathbf{M}_{1 / 2}$ amounts to

$$
\min \left\{\int_{M} d|\nu| \mid \nu \in \mathcal{M}^{n}(M),-\operatorname{div}(\nu)=f^{+}-f^{-}\right\} .
$$

For this different setting, and of course with a different proof, one can look to [33], where this question is formulated in terms of finslerian metric and a careful comparison of these problems on Riemannian manifolds is studied. Another proof is available in [26].

In the next section we will prove that the equivalence $\mathbf{B}=\mathbf{M K}$ still holds for every Lagrangian. However, the equivalence $\mathbf{M}_{p}=\mathbf{M} \mathbf{K}_{p}$ in general fails for $p>\frac{1}{2}$. To explain this phenomenon we prove the following 
Proposition 3.5 For $L(x, v)=g(v, v)^{p}$ with $p>\frac{1}{2}$ it results

$$
\boldsymbol{M}_{p}=\min \left\{M(T)^{2 p} \mid T \in \mathcal{N}_{1}, \partial T=f^{+}-f^{-}\right\} .
$$

Proof. By Theorem 2.4 we have that

$$
\mathbf{M}_{p}=\min \left\{\int_{M}|X(x)|^{2 p} d \sigma \mid T=\sigma \wedge X, \partial T=f^{+}-f^{-}\right\} .
$$

Let $T=\sigma \wedge X$ be optimal in (3.5). Consider the special representation (see (2.1)) with $\left|X_{s}(x)\right|=M(T) \sigma_{s}-$ a.e. By Lemma 2.1 we have $\sigma=c(x) \sigma_{s}$ and $X=\frac{1}{c(x)} X_{s}$ for a positive function $c \in L_{\sigma_{s}}^{1}$ such that $\int_{M} c(x) d \sigma_{s}=1$. Hence

$$
\begin{aligned}
\int_{M}|X(x)|^{2 p} d \sigma & =\int_{M} \frac{1}{c(x)^{2 p}}\left|X_{s}(x)\right|^{2 p} c(x) d \sigma_{s}=M(T)^{2 p} \int_{M} c(x)^{1-2 p} d \sigma_{s} \\
& \geq M(T)^{2 p}\left(\int_{M} c(x) d \sigma_{s}\right)^{1-2 p}=M(T)^{2 p}
\end{aligned}
$$

Where the last inequality follows by the convexity of the function $y \mapsto y^{1-2 p}$ and the Jensen inequality. Then the minimum in (3.5) is exactly $M(T)^{2 p}$.

A consequence of Proposition 3.5 is that $\mathbf{M}_{p}$ is homogeneous with respect to $p$, i.e. for every $\alpha>1$ it results $\mathbf{M}_{\alpha p}=\left(\mathbf{M}_{p}\right)^{\alpha}$. Let us show by a counterexample that this is not the case for $\mathbf{M K}_{p}$. It will follows that the strict inequality $\mathbf{M}_{p}<$ $\mathbf{M K}_{p}$ holds for every $p>\frac{1}{2}$. Indeed, let $T$ be a minimal current for $\mathbf{M} \mathbf{K}_{1 / 2}$. Then, the homogeneity of $\mathbf{M}_{p}$ with respect to $p$ and the equality $\mathbf{M} \mathbf{K}_{p}=\mathbf{M}_{p}$ would imply the following:

$$
\mathbf{M K}_{p}=\mathbf{M}_{p}=M(T)^{2 p}=\mathbf{M K}_{1 / 2}^{2 p} .
$$

However, this equality in general does not hold as we show by the following example.

Example 3.6 Consider the measures $f^{+}=\frac{1}{2} \delta_{x}+\frac{1}{2} \delta_{x^{*}}, f^{-}=\frac{1}{2} \delta_{y}+\frac{1}{2} \delta_{y^{*}}$ in $\mathbb{R}^{2}$ where the points $x, x^{*}, y, y^{*}$ are disposed as in the figure 1 and consider for example the case $p=1$.

An optimal transport plan for both the distance and the distance to the power 2 is given by $\gamma=\frac{1}{2} \delta_{(x, y)}+\frac{1}{2} \delta_{\left(x^{*}, y^{*}\right)}$.

In this case we have

$$
\mathbf{M K}_{1}=\int_{M \times M} d(x, y)^{2} d \gamma(x, y)=\frac{1}{2} d(x, y)^{2}+\frac{1}{2} d\left(x^{*}, y^{*}\right)^{2}=\frac{5}{2} .
$$

On the other hand

$$
\mathbf{M K}_{1 / 2}^{2}=\left(\int_{M \times M} d(x, y) d \gamma(x, y)\right)^{2}=\left(\frac{1}{2} d(x, y)+\frac{1}{2} d\left(x^{*}, y^{*}\right)\right)^{2}=\frac{9}{4} \text {. }
$$

Hence $\mathbf{M K}_{1 / 2}^{2}<\mathbf{M} \mathbf{K}_{1}$. 


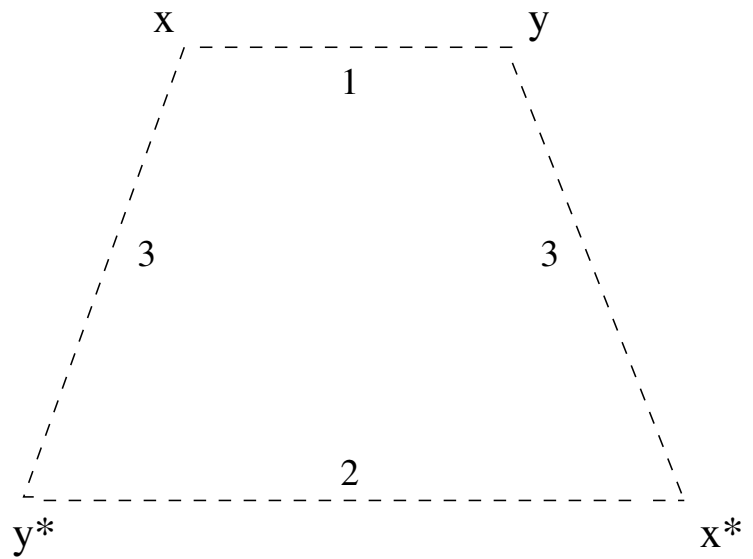

Figure 1 The integer numbers denote the distances between the points.

Let $\gamma$ be an optimal plan for the Monge-Kantorovich problem with the geodesic distance $d$ on $M$ as cost. Consider the 1-current $T_{\gamma}$ defined as follows:

$$
T_{\gamma}(\omega)=\int_{0}^{1} \int_{M \times M}\left\langle\omega\left(\sigma_{x, y}(t)\right), \dot{\sigma}_{x, y}(t)\right\rangle d \gamma(x, y) d t
$$

Where $\sigma_{x, y}$ is a geodesic connecting the points $x, y$. Observe that

$$
\left|T_{\gamma}(\omega)\right| \leq \int_{M \times M} \int_{0}^{1}\left|\dot{\sigma}_{x, y}(t)\right| d t d \gamma(x, y)\|\omega\|_{\infty}=\int_{M \times M} d(x, y) d \gamma(x, y)\|\omega\|_{\infty},
$$

from which we obtain $M\left(T_{\gamma}\right) \leq \mathbf{M K}_{1 / 2}$. By Proposition 3.2 and Proposition 3.5, we have that $T_{\gamma}$ is also optimal for $\mathbf{M}_{p}$ for every $p>\frac{1}{2}$. Furthermore, it results that

$$
M\left(T_{\gamma}\right)=\int_{M \times M} d(x, y) d \gamma(x, y)=\mathbf{M K}_{1 / 2}
$$

Let $\gamma_{p}$ be an optimal plan for $\mathbf{M} \mathbf{K}_{p}$ with $p>\frac{1}{2}$ and let $T_{\gamma_{p}}$ be defined as above. It may happen that the 1-current $T_{\gamma_{p}}$ is not optimal for $\mathbf{M}_{p}$. Indeed, the minimality of $T_{\gamma_{p}}$ also implies that $M\left(T_{\gamma_{p}}\right)=\mathbf{M} \mathbf{K}_{1 / 2}$, and then $\gamma_{p}$ would be an optimal plan also for $\mathbf{M K}_{1 / 2}$. However, it is known that $\mathbf{M} \mathbf{K}_{p}$ admits a unique optimal plan induced by a transport map which in general does not minimize $\mathbf{M K}_{1 / 2}$ (see [23] for more details and explicit examples).

\section{MK as a constrained problem}

In this section we prove that the Monge-Kantorovich problem is in fact equivalent to a refinement of problem $\mathbf{M}$ in which another constraint is added on the 
admissible measures. Actually, the constraint will define a strict subfamily of the admissible measures for $\mathbf{M}$. The result will be achieved in different steps.

\subsection{Duality and Brenier's formulation}

As a first step we establish another duality formula for MK. We have found explicitly this formula in the quadratic case in [32], see also [6]. Furthermore, the strategy to establish this formula is delineated in [9] but in order to include general Lagrangian $L(x, v)$ some additional work is necessary.

Theorem 4.1 The minimum of the Kantorovich problem between $f^{+}$and $f^{-}$is equal to

$\boldsymbol{D}:=\sup _{\phi \in L i p([0,1] \times M)}\left\{\int_{M} \phi(1, x) d f^{-}-\int_{M} \phi(0, x) d f^{+} \mid \partial_{t} \phi+H\left(x, d_{x} \phi\right) \leq 0\right.$ a.e. $\}$.

Proof. Fix $\phi$ admissible for $\mathbf{D}$. For each $(x, y)$ let $\eta$ be an optimal curve for the cost $c(x, y)$ and $\gamma$ an optimal plan for MK. Observe that $\phi(t, \eta(t))$ is absolutely continuous. We have

$$
\begin{aligned}
& \int_{M} \phi(1, x) d f^{-}-\int_{M} \phi(0, x) d f^{+} \\
& \quad=\int_{M \times M} \phi(1, y) d \gamma(x, y)-\int_{M \times M} \phi(0, x) d \gamma(x, y) \\
& \quad=\int_{M \times M} \int_{0}^{1} \frac{d}{d t}[\phi(t, \eta(t))] d t d \gamma(x, y) \\
& \quad=\int_{M \times M} \int_{0}^{1}\left[\partial_{t} \phi(t, \eta(t))+\left\langle d_{x} \phi(t, \eta(t)), \dot{\eta}(t)\right\rangle\right] d t d \gamma(x, y) \\
& \quad \leq \int_{M \times M} \int_{0}^{1}\left[-H\left(\eta(t), d_{x} \phi(t, \eta(t))\right)+\left\langle d_{x} \phi(t, \eta(t)), \dot{\eta}(t)\right\rangle\right] d t d \gamma(x, y) \\
& \quad \leq \int_{M \times M} \int_{0}^{1} L(\eta(t), \dot{\eta}(t)) d t d \gamma(x, y) \\
& \quad=\int_{M \times M} c(x, y) d \gamma(x, y)=\mathbf{M K} .
\end{aligned}
$$

Taking the supremum with respect to $\phi$ we have the inequality $\mathbf{D} \leq \mathbf{M K}$.

Vice versa, using Theorem 2.5, $\forall \varepsilon>0$ we can find continuous functions $f_{\varepsilon}$, $g_{\varepsilon}$ such that

$$
\forall(x, y) \in M \times M: f_{\varepsilon}(x)+g_{\varepsilon}(y) \leq c(x, y)
$$

and such that

$$
\mathbf{M K} \leq \int_{M} f_{\varepsilon} d f^{+}+\int_{M} g_{\varepsilon} d f^{-}+\varepsilon
$$


Consider the Cauchy problem: The function $\phi(t, x)$ defined through the LaxOleinik semigroup by solves this Cauchy problem a.e. Indeed, the general properties of the Lax-Oleinik semigroup (see Theorem 2.7) ensure that $\phi(t, x)$ is a locally Lipschitz function.

If $\left(t_{0}, x_{0}\right)$ is a point of differentiability for $\phi(t, x)$, by Theorem 2.7 there exists an extremal curve $\eta$ with $\eta\left(t_{0}\right)=x_{0}$ such that

$$
\phi\left(t_{0}, x_{0}\right)=-f_{\varepsilon}(\eta(0))+\int_{0}^{t_{0}} L(\eta, \dot{\eta}) d s .
$$

Furthermore, again by Theorem 2.7, we have that $d_{x} \phi\left(t_{0}, x_{0}\right)=\frac{\partial L}{\partial v}\left(\eta\left(t_{0}\right), \dot{\eta}\left(t_{0}\right)\right)$. Observe that the real function

$$
u(t):=\phi(t, \eta(t))+f_{\varepsilon}(\eta(0))-\int_{0}^{t} L(\eta, \dot{\eta}) d s
$$

has a maximum point at $t_{0}$. Hence

$$
\begin{gathered}
0=u^{\prime}\left(t_{0}\right)=\partial_{t} \phi\left(t_{0}, \eta\left(t_{0}\right)\right)+\left\langle d_{x} \phi\left(t_{0}, x_{0}\right), \dot{\eta}\left(t_{0}\right)\right\rangle-L\left(\eta\left(t_{0}\right), \dot{\eta}\left(t_{0}\right)\right)= \\
=\partial_{t} \phi\left(t_{0}, \eta\left(t_{0}\right)\right)+\left\langle\frac{\partial L}{\partial v}\left(\eta\left(t_{0}\right), \dot{\eta}\left(t_{0}\right)\right), \dot{\eta}\left(t_{0}\right)\right\rangle-L\left(\eta\left(t_{0}\right), \dot{\eta}\left(t_{0}\right)\right)= \\
=\partial_{t} \phi\left(t_{0}, x_{0}\right)+H\left(x_{0}, d_{x} \phi\left(t_{0}, x_{0}\right)\right) .
\end{gathered}
$$

Therefore $\phi$ is admissible for $\mathbf{D}$. Furthermore, we remark that $\phi(1, x)=f_{\varepsilon}^{c}(x)$, where $f_{\varepsilon}^{c}(x)$ is the $c$-transform of $f_{\varepsilon}(x)$ defined as in (2.5).

In fact if $y \in M$, and $\eta$ is a Tonelli minimizer for $c(y, x)$, we have

$$
\phi(1, x) \leq-f_{\varepsilon}(y)+\int_{0}^{1} L(\eta, \dot{\eta}) d t=-f_{\varepsilon}(y)+c(y, x) .
$$

Taking the infimum with respect to $y \in M$ we obtain $\phi(1, x) \leq f_{\varepsilon}^{c}(x)$. Vice versa, by Theorem 2.7 there exists a Tonelli minimizer $\eta$ such that

$$
\phi(1, x)=-f_{\varepsilon}(\eta(0))+\int_{0}^{1} L(\eta, \dot{\eta}) d t=-f_{\varepsilon}(\eta(0))+c(\eta(0), x),
$$

from which we obtain

$$
f_{\varepsilon}^{c}(x) \leq \phi(1, x) .
$$

Observe that by (4.1) it results $g_{\varepsilon}(x) \leq f_{\varepsilon}^{c}$, and then by (4.2) we have

$$
\begin{aligned}
\mathbf{M K} & \leq \int_{M} f_{\varepsilon} d f^{+}+\int_{M} g_{\varepsilon} d f^{-}+\varepsilon \leq \int_{M} f_{\varepsilon} d f^{+}+\int_{M} f_{\varepsilon}^{c} d f^{-}+\varepsilon \\
& =\int_{M} \phi(1, x) d f^{-}-\int_{M} \phi(0, x) d f^{+}+\varepsilon \leq \mathbf{D}+\varepsilon .
\end{aligned}
$$

Letting $\varepsilon \rightarrow 0^{+}$we obtain the desired inequality. 


\section{Corollary $4.2 B=M K$.}

Proof. Since $\mathbf{B} \leq \mathbf{M K}$ by Theorem 3.1, it remains to check the opposite inequality. Then fix $\phi$ admissible for $\mathbf{D}$ and $(\rho, E)$ optimal for $\mathbf{B}$. It is possible to show that the curve $t \mapsto \rho_{t}$ is absolutely continuous with respect to the 1Wasserstein distance (see $[1,3,25])$. Therefore, the real function $t \mapsto \int_{M} \phi(t, x) d \rho_{t}$ is absolutely continuous too. Let $\varepsilon>0$ and set $\phi_{\varepsilon}(t, x)=\phi(t, x)-\varepsilon t$, so that $\phi_{\varepsilon} \in \operatorname{Lip}([0,1] \times M)$ and

$$
\partial_{t} \phi_{\varepsilon}+H\left(x, d_{x} \phi_{\varepsilon}\right)=-\varepsilon+\partial_{t} \phi+H\left(x, d_{x} \phi\right) \leq-\varepsilon<0 .
$$

By standard approximation of functions by convolution kernels, we find a sequence of functions $\phi_{n} \in \mathcal{C}^{1}$ admissible for $\mathbf{D}$ and uniformly convergent to $\phi_{\varepsilon}$. We have

$$
\begin{aligned}
- & +\int_{M} \phi(1, x) d f^{-}-\int_{M} \phi(0, x) d f^{+} \\
& =\int_{M} \phi_{\varepsilon}(1, x) d f^{-}-\int_{M} \phi_{\varepsilon}(0, x) d f^{+} \\
& =\lim _{n \rightarrow+\infty}\left(\int_{M} \phi_{n}(1, x) d \rho_{1}-\int_{M} \phi_{n}(0, x) d \rho_{0}\right) \\
& =\lim _{n \rightarrow+\infty} \int_{0}^{1} \frac{d}{d t}\left(\int_{M} \phi_{n}(t, x) d \rho_{t}\right) d t \\
& =\lim _{n \rightarrow+\infty}\left(\int_{0}^{1} \int_{M} \partial_{t} \phi_{n}(t, x) d \rho_{t} d t+\int_{0}^{1} \int_{M} d_{x} \phi_{n}(t, x) \cdot d E_{t} d t\right) \\
& \leq K(\rho, E)=\mathbf{B} .
\end{aligned}
$$

Where we used that $(\rho, E)$ solves the continuity equation, in particular (3.2), in the last equality. In fact, writing

$$
\begin{aligned}
& \frac{1}{h}\left(\int_{M} \phi_{n}(t+h, x) d \rho_{t+h}-\int_{M} \phi_{n}(t, x) d \rho_{t}\right) \\
& \quad=\frac{1}{h}\left(\int_{M}\left(\phi_{n}(t+h, x)-\phi_{n}(t, x)\right) d \rho_{t}+\int_{M} \phi_{n}(t+h, x) d\left(\rho_{t+h}-\rho_{t}\right)\right),
\end{aligned}
$$

we observe that the first term of the above formula yields the first one in (4.3) as $h \rightarrow+\infty$, while the second can be divided in two different terms:

$$
\frac{1}{h} \int_{M} \phi_{n}(t, x) d\left(\rho_{t+h}-\rho_{t}\right)
$$

from which we obtain the second terms of (4.3) because of (3.2), and

$$
\frac{1}{h} \int_{M}\left(\phi_{n}(t+h, x)-\phi_{n}(t, x)\right) d\left(\rho_{t+h}-\rho_{t}\right)
$$


which is negligible since

$$
\frac{1}{h}\left|\int_{M}\left(\phi_{n}(t+h, x)-\phi_{n}(t, x)\right) d\left(\rho_{t+h}-\rho_{t}\right)\right| \leq \operatorname{Lip}\left(\phi_{n}\right) d_{1}\left(\rho_{t+h}, \rho\right) \stackrel{h \rightarrow 0^{+}}{\rightarrow} 0 .
$$

Letting $\varepsilon \rightarrow 0^{+}$we have

$$
\int_{M} \phi(1, x) d f^{-}-\int_{M} \phi(0, x) d f^{+} \leq \mathbf{B} .
$$

Finally, taking the supremum with respect to $\phi$ we obtain $\mathbf{M K} \leq \mathbf{B}$.

Remark 4.3 For the case $L=\varphi(|v|)$ see [9] and [1].

\subsection{Time dependent problem}

As second step we now consider the space $[0,1] \times M$, and indicate with the superscript $^{\sim}$ all the quantity in this product space. In particular $\tilde{v}=\left(v_{0}, v\right) \in \mathbb{R} \times \mathbb{R}^{n}$, and $\tilde{\omega}=\left(\omega_{0}, \omega\right)$. We introduce the new Hamiltonian:

$$
\widetilde{H}(t, x, \tilde{\omega}):=\omega_{0}+H(x, \omega) .
$$

The corresponding Lagrangian is

$$
\tilde{L}(t, x, \tilde{v})=\sup _{\tilde{\omega}}\{\langle\tilde{\omega}, \tilde{v}\rangle-\widetilde{H}(t, x, \tilde{\omega})\}=\sup _{\tilde{\omega}}\left\{\left\langle\omega_{0}, v_{0}\right\rangle+\langle\omega, v\rangle-\omega_{0}-H(x, \omega)\right\} .
$$

From the above definition it follows the relation:

$$
\tilde{L}(t, x, \tilde{v})<+\infty \Leftrightarrow v_{0}=1 \Rightarrow \tilde{L}(t, x, \tilde{v})=L(x, v) .
$$

Define the energy functional:

$$
\widetilde{\mathcal{L}}(\tilde{\sigma}, \widetilde{T})=\sup _{\tilde{\alpha}, \tilde{\omega}}\left\{\int_{0}^{1} \int_{M} \tilde{\alpha}(t, x) d \tilde{\sigma}+\widetilde{T}(\tilde{\omega})\right\} .
$$

Where $\tilde{\sigma} \in \mathcal{P}([0,1] \times M), \widetilde{T} \in \mathcal{N}_{1}([0,1] \times M)$ and the supremum is taken for continuous $(\tilde{\alpha}, \tilde{\omega})$ which satisfy the constraint $\tilde{\alpha}(t, x)+\widetilde{H}(t, x, \tilde{\omega}) \leq 0$.

By (4.4) and Lemma 2.3 we have the following

Lemma 4.4 If $\widetilde{\mathcal{L}}(\tilde{\sigma}, \widetilde{T})<+\infty$ then there exists $\widetilde{X} \in L_{\tilde{\sigma}}^{1}$ such that

$$
\widetilde{T}=\tilde{\sigma} \wedge \tilde{X}, X_{0}(t, x)=1 \quad \tilde{\sigma}-\text { a.e. }
$$

Furthermore, in such case we have that $\widetilde{\mathcal{L}}(\tilde{\sigma}, \widetilde{T})=\int_{0}^{1} \int_{M} L(x, X(t, x)) d \tilde{\sigma}(t, x)$. 
Proof. If $\widetilde{\mathcal{L}}(\tilde{\sigma}, \widetilde{T})<+\infty$ we use the same arguments of Lemma 2.3 in [16] to find $\widetilde{X} \in L_{\tilde{\sigma}}^{1}$ such that: $\widetilde{T}=\tilde{\sigma} \wedge \widetilde{X}$. Let us split $\widetilde{X}=\left(X_{0}, X\right)$.

Let $\omega_{0} \in \mathbb{R}$ and consider the form $\tilde{\omega}=\left(\omega_{0}, \omega\right)$, where $(x, \omega)=\mathcal{L}(x, X(t, x))$ and $\mathcal{L}$ is the Legendre transform on $M$. Then we have

$$
\begin{aligned}
\widetilde{\mathcal{L}}(\tilde{\sigma}, \widetilde{T}) & \geq \int_{0}^{1} \int_{M}-\widetilde{H}(t, x, \tilde{\omega}) d \tilde{\sigma}+\widetilde{T}(\tilde{\omega}) \\
& =-\omega_{0}-\int_{0}^{1} \int_{M} H(x, \omega) d \tilde{\sigma}+\int_{0}^{1} \int_{M}\left\langle\omega_{0}, X_{0}\right\rangle d \tilde{\sigma}+\int_{0}^{1} \int_{M}\langle\omega, X\rangle d \tilde{\sigma} \\
& \left.=\int_{0}^{1} \int_{M} L(x, X(t, x)) d \tilde{\sigma}+\int_{0}^{1} \int_{M}\left\langle\omega_{0},\left(X_{0}(t, x)\right)-1\right)\right\rangle d \tilde{\sigma} .
\end{aligned}
$$

However, by the assumption $\widetilde{\mathcal{L}}(\tilde{\sigma}, \widetilde{T})<+\infty$ we infer that $X_{0}(t, x)=1 \tilde{\sigma}-$ a.e.

The problem in the above argument is that in general $\omega$ is merely Borel. This problem can be solved considering a truncation $\omega_{k}(x)=\omega(x)$ if $|\omega(x)| \leq k$, $\omega_{k}(x)=0$ otherwise, so that $\omega_{k}$ has coefficients in $L_{\sigma}^{\infty}$, and then passing to the limit as $k \rightarrow+\infty$.

Define $F^{+}=\delta_{0} \otimes f^{+}$and $F^{-}=\delta_{1} \otimes f^{-}$and consider the problem:

$$
\widetilde{\mathcal{P}}:=\min \left\{\widetilde{\mathcal{L}}(\tilde{\sigma}, \widetilde{T}) \mid \partial \widetilde{T}=F^{-}-F^{+}\right\},
$$

then the analogous of problem $\mathbf{M}$ on $[0,1] \times M$ is

$$
\widetilde{\mathbf{M}}:=\min \left\{\int_{T([0,1] \times M)} \tilde{L}(t, x, \tilde{v}) d \tilde{\mu} \mid \tilde{\mu} \in \mathcal{P}(T([0,1] \times M)), \partial p(\tilde{\mu})=F^{-}-F^{+}\right\} .
$$

We have the following relationship with the Monge-Kantorovich problem.

Theorem $4.5 M K=\widetilde{\mathcal{P}}=\widetilde{M}$.

Proof. If $\widetilde{T}$ is admissible for $\widetilde{\mathcal{P}}$, by Theorem 4.1 it results

$$
\mathbf{M K}=\sup _{\phi \in \operatorname{Lip}([0,1] \times M)}\left\{\langle\widetilde{T}, d \phi\rangle \mid \partial_{t} \phi+H\left(x, d_{x} \phi\right) \leq 0\right\} .
$$

Hence, for any regular function $\phi(t, x)$ the couple $(0, d \phi)$ is admissible for $(4.5)$ and then $\mathbf{M K} \leq \widetilde{\mathcal{P}}$.

Vice versa, consider $\tilde{\gamma}$ optimal plan between $F^{+}$and $F^{-}, \tilde{\eta}$ optimal curve for the cost $\tilde{c}(a, b)$ associated to $\tilde{L}$ defined as in (1.2), and denote by $\widetilde{\mathbf{M K}}$ the corresponding mass transportation problem. Furthermore consider the measure $\tilde{\sigma}$ defined as follows:

$$
\int_{0}^{1} \int_{M} f(t, x) d \tilde{\sigma}=\int_{([0,1] \times M)^{2}} \int_{0}^{1} f(\tilde{\eta}(t)) d t d \tilde{\gamma}(a, b) .
$$


Also define:

$$
\widetilde{T}(\tilde{\omega})=\int_{([0,1] \times M)^{2}} \int_{0}^{1}\langle\tilde{\omega}(\tilde{\eta}(t)), \dot{\tilde{\eta}}(t)\rangle d t d \tilde{\gamma}(a, b) .
$$

Then

$$
\begin{aligned}
\widetilde{T}(d \phi) & =\int_{([0,1] \times M)^{2}} \int_{0}^{1}\langle d \phi(\tilde{\eta}(t)), \dot{\tilde{\eta}}(t)\rangle d t d \tilde{\gamma}(a, b) \\
& =\int_{([0,1] \times M)^{2}} \int_{0}^{1} \frac{d}{d t}[\phi(\tilde{\eta}(t))] d t d \tilde{\gamma}(a, b) \\
& =\int_{([0,1] \times M)^{2}}[\phi(b)-\phi(a)] d \tilde{\gamma}(a, b) \\
& =\int_{[0,1] \times M} \phi(b) d F^{-}-\int_{[0,1] \times M} \phi(a) d F^{+} .
\end{aligned}
$$

And then $\partial \widetilde{T}=F^{-}-F^{+}$.

Hence, if $(\tilde{\alpha}, \tilde{\omega})$ is admissible for (4.5), we have

$$
\begin{aligned}
\int_{0}^{1} & \int_{M} \tilde{\alpha}(t, x) d \tilde{\sigma}+\tilde{T}(\tilde{\omega}) \\
& =\int_{([0,1] \times M)^{2}} \int_{0}^{1}[\tilde{\alpha}(\tilde{\eta}(t))+\langle\tilde{\omega}(\tilde{\eta}(t)), \dot{\tilde{\eta}}(t)\rangle] d t d \tilde{\gamma}(a, b) \\
& \leq \int_{([0,1] \times M)^{2}} \int_{0}^{1}[-\tilde{H}(\tilde{\eta}(t), \tilde{\omega}(\tilde{\eta}(t)))+\langle\tilde{\omega}(\tilde{\eta}(t)), \dot{\tilde{\eta}}(t)\rangle] d t d \tilde{\gamma}(a, b) \\
& \leq \int_{([0,1] \times M)^{2}} \int_{0}^{1} \tilde{L}(\tilde{\eta}, \dot{\tilde{\eta}}) d t d \tilde{\gamma}(a, b) \\
& =\int_{([0,1] \times M)^{2}} \tilde{c}(a, b) d \tilde{\gamma}(a, b)=\widetilde{\mathbf{M K}}
\end{aligned}
$$

Taking the supremum with respect to $(\tilde{\alpha}, \tilde{\omega})$ we obtain $\widetilde{\mathcal{P}} \leq \widetilde{\mathbf{M K}}$. Now, let us take $\gamma$ an optimal plan on $M \times M$ between $f^{+}$and $f^{-}$and consider the plan between $F^{+}$and $F^{-}$defined as follows:

$$
\tilde{\gamma}=\delta_{0}\left(t_{1}\right) \otimes \delta_{1}\left(t_{2}\right) \otimes \gamma
$$

Let $\eta$ be an optimal curve for the cost $c\left(x_{1}, x_{2}\right)$ on $M$, and considering the curve $\tilde{\eta}(t)=(t, \eta(t))$ on $[0,1] \times M$ we can evaluate

$$
\begin{aligned}
\widetilde{\mathcal{P}} \leq \widetilde{\mathbf{M K}} & \leq \int_{([0,1] \times M)^{2}} \tilde{c}\left(t_{1}, x_{1}, t_{2}, x_{2}\right) d \tilde{\gamma}\left(t_{1}, x_{1}, t_{2}, x_{2}\right) \\
& =\int_{M \times M} \tilde{c}\left(0, x_{1}, 1, x_{2}\right) d \gamma\left(x_{1}, x_{2}\right)
\end{aligned}
$$




$$
\begin{aligned}
& \leq \int_{M \times M} \int_{0}^{1} \tilde{L}(\tilde{\eta}, \dot{\tilde{\eta}}) d t d \gamma\left(x_{1}, x_{2}\right) \\
& =\int_{M \times M} \int_{0}^{1} L(\eta, \dot{\eta}) d t d \gamma\left(x_{1}, x_{2}\right) \\
& =\int_{M \times M} c\left(x_{1}, x_{2}\right) d \gamma\left(x_{1}, x_{2}\right)=\mathbf{M K},
\end{aligned}
$$

which conclude the proof of the first equivalence.

To prove the second equivalence let us consider $\tilde{\mu}$ admissible for $\widetilde{\mathbf{M}}$. By Disintegration Theorem 2.8 we can write $\tilde{\mu}=\tilde{\nu}_{t, x} \otimes \tilde{\sigma}$, with

$$
\tilde{\nu}_{t, x} \in \mathcal{P}\left(T_{(t, x)}([0,1] \times M)\right), \quad \tilde{\sigma} \in \mathcal{P}([0,1] \times M) .
$$

Using Jensen's inequality we have

$$
\begin{aligned}
\widetilde{\mathcal{L}}(\tilde{\sigma}, p(\tilde{\mu})) & \leq \int_{[0,1] \times M} \tilde{L}\left(t, x, \int_{T_{(t, x)}([0,1] \times M)} \tilde{v} d \tilde{\nu}_{t, x}\right) d \tilde{\sigma} \\
& \leq \int_{[0,1] \times M} \int_{T_{(t, x)}([0,1] \times M)} \tilde{L}(t, x, \tilde{v}) d \tilde{\nu}_{t, x} d \tilde{\sigma} \\
& =\int_{T([0,1] \times M)} \tilde{L}(t, x, \tilde{v}) d \tilde{\mu}=\mathcal{A}(\tilde{\mu}) .
\end{aligned}
$$

Then $\widetilde{\mathcal{P}} \leq \widetilde{\mathbf{M}}$. Vice versa, let us take $(\tilde{\sigma}, \widetilde{T})$ such that $\tilde{\mathcal{L}}(\tilde{\sigma}, \widetilde{T})<+\infty$. By Lemma 4.4 we have that $\widetilde{T}=\tilde{\sigma} \wedge \widetilde{X}$ with $X_{0}(t, x)=1 \tilde{\sigma}$ - a.e.

Considering $i(\tilde{\sigma}, \widetilde{T})=(i d \times \widetilde{X})_{\sharp} \tilde{\sigma}$ we obtain

$$
\mathcal{A}(i(\tilde{\sigma}, \widetilde{T}))=\int_{[0,1] \times M} \tilde{L}(t, x, \widetilde{X}) d \tilde{\sigma}=\int_{[0,1] \times M} L(x, X(t, x)) d \tilde{\sigma}=\widetilde{\mathcal{L}}(\tilde{\sigma}, \widetilde{T}) .
$$

Then $\widetilde{\mathbf{M}} \leq \widetilde{\mathcal{P}}$.

Remark 4.6 It is not difficult to check that $\widetilde{\mathcal{P}}$ admits minimizers (see [16]). Then the arguments above tells us that $\widetilde{\mathbf{M}}$ admits minimizers too.

\subsection{The additional constraint}

In the previous subsection we proved that the Monge-Kantorovich problem is equivalent to the problem

$$
\widetilde{\mathbf{M}}=\min \left\{\int_{T([0,1] \times M)} \tilde{L}(t, x, \tilde{v}) d \tilde{\mu} \mid \tilde{\mu} \in \mathcal{P}(T([0,1] \times M)), \partial p(\tilde{\mu})=F^{-}-F^{+}\right\} .
$$


However, by (4.4) we know that $\tilde{L}(t, x, \tilde{v})<+\infty \Leftrightarrow v_{0}=1$. Hence, the value of the minimum does not change if we minimize on the set of measures of the form $\tilde{\mu}=\delta_{1} \otimes \bar{\mu}$, with $\bar{\mu} \in \mathcal{M}([0,1] \times T M)$.

Considering the projection on the second factor $\pi_{(x, v)}:[0,1] \times T M \rightarrow T M$ and $\mu=\left(\pi_{(x, v)}\right)_{\sharp} \bar{\mu}$, by Disintegration Theorem 2.8 there exists $\nu_{x, v} \in \mathcal{P}([0,1])$ such that $\bar{\mu}=\nu_{x, v} \otimes \mu$. Hence we have

$$
\begin{aligned}
\int_{T([0,1] \times M)} \tilde{L}(t, x, \tilde{v}) d \tilde{\mu} & =\int_{0}^{1} \int_{T M} L(x, v) d \bar{\mu} \\
& =\int_{T M}\left(\int_{0}^{1} L(x, v) d \nu_{x, v}(t)\right) d \mu(x, v) \\
& =\int_{T M} L(x, v) d \mu(x, v) .
\end{aligned}
$$

Now we compute the boundary of $p(\mu)$. First, observe that

$$
\begin{gathered}
d_{(t, x)} \varphi\left(\pi_{(x, v)}(t, x, v)\right) \\
=\partial_{t} \varphi\left(\pi_{(x, v)}(t, x, v)\right) \partial_{t}\left(\pi_{(x, v)}(t, x, v)\right)+d_{x} \varphi\left(\pi_{(x, v)}(t, x, v)\right) \cdot \partial_{x}\left(\pi_{(x, v)}(t, x, v)\right) \\
=d_{x} \varphi\left(\pi_{(x, v)}(t, x, v)\right) .
\end{gathered}
$$

Then we can evaluate

$$
\begin{aligned}
\int_{T M}\langle d \varphi, v\rangle d \mu & =\int_{0}^{1} \int_{T M}\left\langle d_{x} \varphi\left(\pi_{(x, v)}(t, x, v)\right), v\right\rangle d \bar{\mu} \\
& =\int_{T([0,1] \times M)}\left\langle d_{(t, x)} \varphi\left(\pi_{(x, v)}(t, x, v)\right), \tilde{v}\right\rangle d \tilde{\mu} \\
& =\int_{0}^{1} \int_{M} \varphi\left(\pi_{(x, v)}(t, x, v)\right) d\left(F^{-}-F^{+}\right) \\
& =\int_{M} \varphi(x) d f^{-}-\int_{M} \varphi(x) d f^{+} \Leftrightarrow \partial p(\mu)=f^{-}-f^{+} .
\end{aligned}
$$

Then we define

$$
\Lambda=\left\{\mu \in \mathcal{P}(T M) \mid \exists \nu_{x, v} \in \mathcal{P}([0,1]) \text { s.t. } \partial p\left(\delta_{1} \otimes \nu_{x, v} \otimes d \mu\right)=F^{-}-F^{+}\right\},
$$

where the family of measuures $\nu_{x, v} \in \mathcal{P}([0,1])$ is defined for $\mu$-a.e. $(x, v) \in T M$ and writing $\delta_{1} \otimes \nu_{x, v} \otimes d \mu$ we assume that $\nu_{x, v}$ satisfy the properties (i) and (ii) of Theorem 2.8. Then, consider the problem

$$
\mathbf{M}_{c}:=\min \{\mathcal{A}(\mu) \mid \mu \in \Lambda\} .
$$

By uniqueness of disintegration of measures and Theorem 4.5 we can state the following 
Theorem 4.7 $M K=M_{c}$.

Therefore, the Monge-Kantorovich problem can be obtained from problem $\mathbf{M}$ on the phase space by adding a constraint on the set of the admissible measures. This additional constraint actually define a strict subfamily of admissible measures as we are going to prove.

By definition of $F^{-}, F^{+}$, a measure $\mu \in \Lambda$ if and only if

$$
\int_{T M} \int_{0}^{1}\left[\partial_{t} \varphi+\left\langle d_{x} \varphi, v\right\rangle\right] d \nu_{x, v}(t) d \mu(x, v)=\int_{M} \varphi(1, x) d f^{-}-\int_{M} \varphi(0, x) d f^{+},
$$

for every regular function $\varphi(t, x)$ on $[0,1] \times M$.

Lemma 4.8 Let $f:[0,1] \rightarrow \mathbb{R}$ be a continuous function. Then

$$
\int_{T M}\left(\int_{0}^{1} f(t) d \nu_{x, v}(t)\right) d \mu(x, v)=\int_{0}^{1} f(t) d t .
$$

Proof. It is sufficient to take $F(t)=\int_{0}^{t} f(s) d s$ and to put $\varphi(t, x)=F(t)$ into (4.8).

Observe that if $\mu \in \Lambda$ is such that the disintegration $\nu_{x, v}$ is independent from $(x, v)$, then by Lemma $4.8 \nu_{x, v}=\mathcal{L}_{[[0,1]}^{1}$. The following example shows that there are admissible measures $\mu$ for $\mathbf{M}$ which do not belongs to $\Lambda$. Furthermore, we will prove that in the case of $f^{+}=f^{-}$every such measure $\mu$ belongs to $\Lambda$. Actually, it turns out that the Lebesgue measure on $[0,1]$ always satisfy the condition (4.8) in place of $\nu_{x, v}$ in the case of $f^{+}=f^{-}$, while it in general fails for $f^{+} \neq f^{-}$. Note that in our case the choice $f^{+}=f^{-}$is non-trivial and corresponds to minimize the action of closed measures as in Mather's problem $\mathbf{P}$. For sake of simplicity, in the following examples we consider $M=[0,1]$. However, the same arguments can be repeated on $S^{1}$ without further difficulties.

Example 4.9 Let $M=[0,1], \mu=2 x \mathcal{L}_{[[0,1]}^{1} \otimes \delta_{\frac{1}{2}}$ and suppose that $\mu \in \Lambda$. Observe that

$$
\begin{gathered}
\int_{T M}\langle d \varphi, v\rangle d \mu=\int_{0}^{1} \varphi^{\prime}(x) x d x \\
=\varphi(1)-\int_{0}^{1} \varphi(x) d x \Rightarrow \partial p(\mu)=\delta_{1}-\mathcal{L}_{[[0,1]}^{1} .
\end{gathered}
$$

Putting $\varphi(t, x)=t f(x)$ into (4.8) we obtain

$$
\int_{0}^{1} 2 x f(x) d x+\int_{0}^{1}\left(\int_{0}^{1} t d \nu_{x, \frac{1}{2}}(t)\right) f^{\prime}(x) x d x=f(1) .
$$


Taking $f(x)=2 x$, by Lemma 4.8 the left side of (4.9) becomes

$$
\int_{0}^{1} 4 x^{2} d x+\int_{0}^{1} t d t=\frac{11}{6} .
$$

However, this is a contradiction since the right side of (4.9) is equal to 2.

The previous example can be repeated on $S^{1}$ with the same technique taking the measures $\mu_{\varepsilon}=\frac{x-\varepsilon}{c_{\varepsilon}} \mathcal{L}_{[[\varepsilon, 1-\varepsilon]}^{1} \otimes \delta_{c_{\varepsilon}}$, with $c_{\varepsilon}=\frac{1}{2}+2 \varepsilon(\varepsilon-1)$, and then letting $\varepsilon \rightarrow 0^{+}$.

We also remark that in the case $L(x, v)=g(v, v)^{\frac{p}{2}}$ for $p>1$, the values of the functionals in $\mathbf{M}_{2 p}$ and $\mathbf{M K}_{2 p}$ satisfy the relation

$$
\mathbf{M}_{2 p}=\int_{T M}|v|^{p} d \mu=\frac{1}{2^{p}}<\frac{1}{p+1}=\int_{M}|x|^{p} d x=d_{p}\left(\mathcal{L}_{[[0,1]}^{1}, \delta_{1}\right)^{p}=\mathbf{M K}_{2 p},
$$

showing the necessity of the additional constraint $\Lambda$ in (4.7) to establish the equality $\mathbf{M K}=\mathbf{M}_{c}$.

However, if $f^{+}=f^{-}$then the additional constraint $\Lambda$ in $\mathbf{M}_{c}$ does not play any role, as results by the following

Lemma 4.10 If $f^{+}=f^{-}$then every closed measures $\mu \in \mathcal{P}(T M)$ belongs to $\Lambda$.

Proof. Let $\mu \in \mathcal{P}(T M)$ be a closed measure. By (1.5) and (1.4) this is equivalent to have $\partial p(\mu)=0=\sigma-\sigma$, with $\sigma$ the projection of $\mu$ on $M$. Taking $\tilde{\mu}=$ $\delta_{1} \otimes \mathcal{L}_{[[0,1]}^{1} \otimes \mu$, for every regular function $\varphi(t, x)$ on $[0,1] \times M$ we have

$$
\begin{aligned}
& \int_{T([0,1] \times M)}\langle d \varphi(t, x), \tilde{v}\rangle d \tilde{\mu} \\
& \quad=\int_{T M}\left(\int_{0}^{1} \partial_{t} \varphi(t, x) d t\right) d \mu(x, v)+\int_{T M}\left(\int_{0}^{1}\left\langle d_{x} \varphi(t, x), v\right\rangle d t\right) d \mu(x, v) \\
& \quad=\int_{T M}(\varphi(1, x)-\varphi(0, x)) d \mu(x, v)=\int_{M}\left(\int_{T_{x} M}(\varphi(1, x)-\varphi(0, x)) d \nu_{x}(v)\right) d \sigma \\
& \quad=\int_{M} \varphi(1, x) d \sigma-\int_{M} \varphi(0, x) d \sigma \Rightarrow \partial p(\tilde{\mu})=F^{-}-F^{+},
\end{aligned}
$$

where the second addendum in the second equality is zero since $\partial p(\mu)=0$, and we have again used the Disintegration Theorem 2.8. Hence $\mu \in \Lambda$.

Denoted with $\mathbf{M}_{c}^{0}$ the value of $\mathbf{M}_{c}$ in the case of $f^{+}=f^{-}$, as consequence of Lemma 4.10 we have the following

Proposition $4.11-c_{0}:=\min \{\mathcal{A}(\mu) \mid \partial p(\mu)=0\}=\boldsymbol{M}_{c}^{0}$. 
Where $c_{0}$ is known as the Mañé critical value (see [15, 20, 21]). We have the same result if we also consider an homological constraint and then recovering Mather's problem $\mathbf{P}$.

We conclude showing that in the case of $f^{+} \neq f^{-}$the Lebesgue measure in general does not satisfy the condition (4.8) in place of $\nu_{x, v}$.

Example 4.12 Let again $M=[0,1]$, and $\mu=\mathcal{L}_{[[0,1]}^{1} \otimes \delta_{1}$. Observe that

$$
\begin{aligned}
& \int_{T M}\langle d \varphi, v\rangle d \mu=\int_{0}^{1} \varphi^{\prime}(x) d x \\
= & \varphi(1)-\varphi(0) \Rightarrow \partial p(\mu)=\delta_{1}-\delta_{0} .
\end{aligned}
$$

Now, putting $\varphi(t, x)=t f(x)$ and $\nu_{x, v}(t)=\mathcal{L}_{[[0,1]}^{1}$ into (4.8) we obtain

$$
\begin{array}{r}
\int_{0}^{1} f(x) d x+\int_{0}^{1}\left(\int_{0}^{1} t d t\right) f^{\prime}(x) d x=f(1) \\
\Leftrightarrow \int_{0}^{1} f(x) d x+\frac{1}{2} \int_{0}^{1} f^{\prime}(x) d x=f(1) \\
\Leftrightarrow \int_{0}^{1} f(x) d x=\frac{1}{2} f(0)+\frac{1}{2} f(1) .
\end{array}
$$

Any regular function $f$ with zero boundary values and non-vanishing integral is a counterexample to (4.10).

In this particular case we can take $\nu_{x}(t)=\delta_{x}(t)$ to obtain

$$
\begin{aligned}
\int_{0}^{1} & \int_{0}^{1} \partial_{t} \varphi(t, x) d \nu_{x}(t) d x+\int_{0}^{1} \int_{0}^{1} \partial_{x} \varphi(t, x) d \nu_{x}(t) d x \\
& =\int_{0}^{1} \partial_{t} \varphi(x, x) d x+\int_{0}^{1} \partial_{x} \varphi(x, x) d x \\
& =\int_{0}^{1} \frac{d}{d x} \varphi(x, x) d x=\varphi(1,1)-\varphi(0,0),
\end{aligned}
$$

and then satisfying the condition (4.8).

The previous example can be repeated on $S^{1}$ taking the measure $\mu_{\varepsilon}=\frac{1}{1-2 \varepsilon} \mathcal{L}^{1}\left\llcorner[\varepsilon, 1-\varepsilon] \otimes \delta_{1}\right.$.

\section{Acknowledgments}

The author is grateful to professor G. Buttazzo and L. De Pascale for proposing this problem and for the constant attention.

Note. Some months after this paper was written we have been informed of the existence of the paper [6] of Bernard and Buffoni which also deals with the 
connections between optimal mass transportation problems and Mather theory, addressing some questions related to those studied in this paper. In fact, in that paper the authors exploit the instruments of the weak KAM theory of Albert Fathi to introduce transport problem related to a generic Lagrangian (even time depending) and to relate this transport problems to the Mather's one.

\section{References}

[1] L. AMBROSIO, Lecture Notes on Transport Problems, in Mathematical Aspects of Evolving Interfaces, Lecture Notes in Mathematics 1812, Springer, Berlin, 2003, 1-52.

[2] L. AMBrosiO, N. FUSCO and D. PALlARA, Functions of Bounded Variation and Free Discontinuity Problems, Oxford University Press, New York, 2000 .

[3] L. AMBrosio, N. GIGLI and G. SAVARÉ, Gradient Flows in Metric Spaces and the Wasserstein Spaces of Probability Measures, Lectures in Mathematics, ETH Zurich, Birkhäuser, 2005.

[4] V. BANGERT, Minimal measures and minimizing closed normal onecurrents, GAFA Geom. Funct. Anal. 9(3) (1999), 413-427.

[5] J. BENAMOU and Y. BRENIER, A computational fluid mechanics solution to the Monge-Kantorovich mass transfer problem, Numer. Math. 84(3) (2000), 375-393.

[6] P. BERNARD and B. BUFFONI, Optimal mass transportation and Mather theory, to appear on Journal European Math. Soc.

[7] G. BOUCHITTÉ and G. BUTTAZZO, Characterization of optimal shapes and masses through Monge-Kantorovich equation, Journal European Math. Soc. 3 (2001), 139-168.

[8] G. BOUCHITTÉ, G. BUTTAZZO and P. SEPPECHER, Shape optimization solutions via Monge-Kantorovich equation, C. R. Acad. Sci. Paris 324-I (1997), 1185-1191.

[9] Y. BRENIER, Extended Monge-Kantorovich Theory, in Optimal Transportation and Applications, Lecture Notes in Mathematics 1813, Springer, Berlin, 2003, 91-121.

[10] Y. BRENIER, Dcomposition polaire et rarrangement des champs des vecteurs, C. R. Acad. Sci. Paris. Sr. I Math. 305(19) (1987), 805-808.

[11] Y. BRENIER, Polar factorization and monotone rearrangement of vectorvalued functions, Comm. Pure Appl. Math. 44(4) (1991), 375-417. 
[12] Y. BRENIER, A homogenized model for vector sheets, Arch. Rat. Mech. Anal. 138 (1997), 319-353.

[13] Y. BRENIER, A Monge-Kantorovich Approach to the Maxwell Equations, Hyperbolic Problems: Theory, Numerics, Applications, Vol. I, II, Internat. Ser. Numer. Math. 140, 141, Birkhäuser, Basel, 2001, 179-186.

[14] Y. BRENIER, Extension of the Monge-Kantorovich Theory to Classical Electrodynamics, Recent advances in the theory and applications of mass transport, Contemp. Math., 353, AMS, Providence, RI, 2004, 19-41.

[15] G. CONTRERAS and R. ITURRIAGA, Global Minimizers of Autonomous Lagrangians, Coloquio Brasileiro de Matematica, IMPA, Rio de Janeiro, 1999.

[16] L. DE PASCALE, M. GELLI and L. GRANIERI, Minimal measures, onedimensional currents and the Monge-Kantorovich problem, to appear on Calc. Var.

[17] L. C. EVANS, Some new PDE methods for weak KAM theory, Calc. Var. Partial Differential Equations 17(2) (2003), 159-177.

[18] L. C. EVANS, Partial differential equations and Monge-Kantorovich mass transfer (survey paper). Current Developments in Mathematics 1997, International Press (1999), edited by S. T. Yau.

[19] L. C. EVANS and D. GOMES, Linear programming interpretation of Mather's variational principle, ESAIM Control Optim. Calc. Var. 8 (2002), 693-702.

[20] A. FATHI, Weak KAM Theorem in Lagrangian Dynamics, forthcoming book, Cambridge University Press.

[21] A. FATHI and A. SICONOLFI, Existence of $C^{1}$ critical subsolutions of the Hamilton-Jacobi equation, Invent. Math. 155(2) (2004), 363-388.

[22] H. FEDERER, Geometric Measure Theory, Springer, Berlin, 1969.

[23] W. GANGBO and R. J. MCCANN, The geometry of optimal transportation, Acta Math. 177 (1996), 113-161.

[24] M. GIAQUINTA, G. MODICA and J. SOUČEK, Cartesian Currents in the Calculus of Variations. I. Cartesian currents, Ergebnisse der Mathematik und ihrer Grenzgebiete. 3. Folge, A Series of Modern Surveys in Mathematics 37, Springer-Verlag, Berlin, 1998.

[25] L. GRANIERI, Mass Transportation Problems and Minimal Measures, Ph.D. Thesis in Mathematics, Pisa, 2005. 
[26] S. HOHLOCH, Optimale Massebewegung im Monge-Kantorovich Transport Problem, Diplomarbeit, Dezember 2002.

[27] R. JORDAN, D. KINDERLEHRER and F. OTTO, The variational formulation of the Fokker-Plank equation, Siam J. Math. Anal. 29 (1998), 1-17.

[28] R. MAÑÉ, Generic properties and problems of minimizing measures of Lagrangian systems, Nonlinearity 9(2) (1996), 273-310.

[29] J. MATHER, Action minimizing invariant measures for positive definite Lagrangian systems, Math. Z. 207(2) (1991), 169-207.

[30] J. MATHER, Minimal measures, Comment. Math. Helv. 64(3) (1989), 375394.

[31] F. OTTO, The geometry of dissipative evolution equations: the porus medium equation, Comm. Partial Differential Equations 26(1-2) (2001) 101174

[32] F. OTTO and C. VILLANI, Generalization of an inequality by Talagrand and links with the logaritmic Sobolev inequality, Journal of Functional Analysis 173 (2000), 361-400.

[33] A. PRATELLI, Equivalence between some definitions for the optimal mass transport problem and for the transport density on manifolds, Ann. Mat. Pura Appl. (4) 184(2) (2005), 215-238.

[34] S. T. RACHEV and L. RUSCHENDORF, Mass Transportation Problems, Probab. Appl., Springer-Verlag, New York, 1998.

[35] C. VILLANI, Topics in Optimal Transportation, Graduate Studies in mathematics 58, AMS, Providence, RI, 2003.

Received 16 March 2005; accepted 15 October 2005

Published Online First 13 August 2007 